To be published in The Outer Heliosphere: The Next Frontiers - Proceedings of a COSPAR Colloquium held in Potsdam, Germany, July 24-28, 2000.

\title{
Scientific Payload for an Interstellar Probe Mission
}

\author{
R. A. Mewaldt ${ }^{1}$, P. C. Liewer ${ }^{2}$, and the Interstellar Probe Science and Technology Definition \\ Team* \\ ${ }^{1}$ California Institute of Technology, Pasadena, CA 91125 \\ ${ }^{2}$ Jet Propulsion Laboratory, California Institute of Technology, Pasadena, CA 91109
}

NASA's Interstellar Probe Mission will be the first spacecraft specifically designed to explore the outer solar system, pass through the boundaries of the heliosphere, and sample the nearby interstellar medium. During the spring of 1999, NASA's Interstellar Probe Science and Technology Definition Team* developed a concept for a mission that will travel to 200400 AU using solar-sail propulsion. The principal scientific goals would be to explore the outer solar system, explore the structure of the heliosphere and its interaction with the interstellar medium, and explore the nature of the interstellar medium itself. These studies would be carried out by a $\sim 150 \mathrm{~kg}$ spacecraft carrying a scientific payload designed to make comprehensive measurements of heliospheric and interstellar plasma, fields, energetic particles, neutral gas, and dust. We discuss the scientific goals and strawman payload for this mission.

\section{INTRODUCTION}

The rapidly expanding solar atmosphere - the solar wind - creates a large bubble called the heliosphere that shields our solar system from the interstellar plasma and magnetic fields, and most of the cosmic rays and dust that comprise the local galactic neighborhood (Figure 1). Outside of this bubble is a new, unexplored region about which we know very little. The Interstellar Probe mission is designed to exit this bubble and begin exploring the space between the stars. In the course of this journey, Interstellar Probe will investigate unknown aspects of the outer solar system, explore the boundaries of the heliosphere to reveal how a star interacts with its environment, and directly sample the properties of the nearby interstellar medium (ISM). These studies will address key questions about the nature of the primordial solar nebula, the structure and dynamics of our heliosphere, the properties of material in the

\footnotetext{
* Interstellar Probe Science and Technology Definition Team (ISPSTDT). Chairman: R. Mewaldt, Caltech; Study Scientist: P. Liewer, JPL. Team Members: E. Bakes, NASA Ames; P. Frisch, U. of Chicago; H. Funsten, LANL; M. Gruntman, USC; L. Johnson, MSFC; R. Jokipii, U. of Arizona; W. Kurth, U. of Iowa; J. Linsky, U. of Colorado; R. Malhotra, NASA LPI; I. Mann, Caltech; R. McNutt, APL; E. Moebius, UNH; W. Reach, Caltech; S. Suess, MSFC; A. Szabo, GSFC; J. Trainor, GSFC/retired; G. Zank, Bartol; T. Zurbuchen, U. of Michigan. Program Manager: S. Gavit, JPL. Program Scientist: V. Jones, NASA HQ. Deputy Program Scientist: J. Ling, NASA HQ. Program Executive: G. Mucklow, NASA HQ. NASA Transportation: D. Stone, NASA HQ. Interagency Representatives: D. Goodwin, DOE and E. Loh, NSF. Foreign Guest Participants: B. Heber, Max Planck Inst., Germany; C. Maccone, Torino, Italy. JPL Support: J. Ayon, E. De Jong, N. Murphy, R. Wallace.
} 


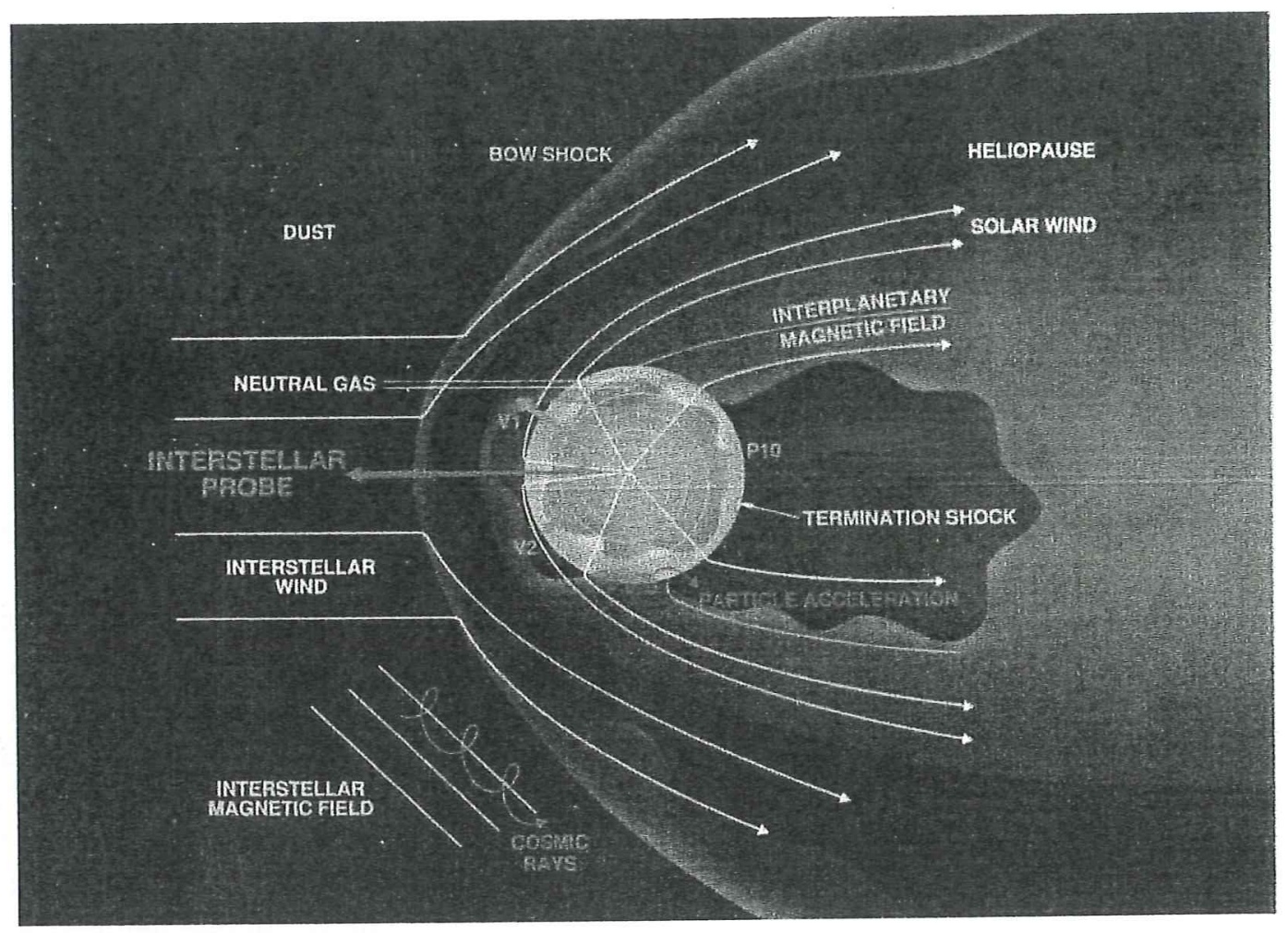

FIG. 1: Illustration of the structure of the heliosphere created when the supersonic solar wind diverts the interstellar flow around the Sun. Interstellar ions and neutrals flow at $26 \mathrm{~km} / \mathrm{s}$ relative to the Sun. The solar wind, flowing outward at $400-800 \mathrm{~km} / \mathrm{s}$, makes a transition to subsonic flow at the termination shock, beyond which it is turned toward the heliotail, carrying along the interplanetary magnetic field. The heliopause separates solar material and magnetic fields from interstellar material and fields. There may also be a bow shock beyond the heliopause. Of the spacecraft shown, only Voyager $1 \& 2$ still operate.

outer solar system, the nature of other stellar systems, the chemical evolution of our Galaxy, and the origins of matter in the earliest days of our universe. To carry out these exploratory studies Interstellar Probe will include a suite of sensors designed to measure the detailed properties of the plasma, neutral atoms, energetic particle, magnetic fields, and dust in the outer heliosphere and nearby ISM. A 400-m diameter solar sail would accelerate the spacecraft to $\sim 15 \mathrm{AU} /$ year, roughly four times the speed of Voyager $1 \& 2$. Recent estimates place the termination shock at 80 to $100 \mathrm{AU}$ from the Sun, with the heliopause at 120 to 150 AU (see, e.g., Stone and Cummings 1999). Interstellar Probe would be designed to reach a minimum of $200 \mathrm{AU}$ within 15 years, with sufficient consumables to last to $400 \mathrm{AU}$.

The scientific importance of sending a spacecraft through the boundaries of the heliosphere into nearby interstellar space has been recognized by a number of studies by the National Academy of Science (e.g., Scarf 1988; Burke 1988; Neugebauer 1995) and NASA Roadmaps (e.g., Burch et al. 1996, Strong et al. 1999). Several previous mission concepts with related goals have been studied (e.g., Jaffe et al. 1977, Jaffe and Ivie 1979; Etchegaray 1987, Nock 1988, Holzer et al. 1990; Mewaldt et al. 1995), most of which have relied on rather large spacecraft. Recent advances now make it feasible to accomplish this mission using a solar sail to accelerate $\mathrm{a} \sim 150 \mathrm{~kg}$ spacecraft. 
The mission concept presented here was formulated by the Interstellar Probe Science and Technology Definition Team (ISPSTDT), sponsored by the NASA Office of Space Science (OSS). Their primary goal was to develop a mission concept for the Sun-Earth-Connection Roadmap (http://www.lmsal.com/sec), as part of NASA's strategic planning activities. As a result of these activities an interstellar probe mission is now included in the new Space Science Enterprise Strategic Plan. A summary of the science goals and mission concept can be found at http://interstellar.jpl.nasa.gov.

\section{SCIENCE OBJECTIVES}

Interstellar Probe's unique voyage from Earth to beyond 200 AU will enable the first comprehensive measurements of plasma, neutrals, dust, magnetic fields, energetic particles, cosmic rays, and infrared emission from the outer solar system, though the boundaries of the heliosphere, and on into the very local interstellar medium (VLISM). This will allow the mission to address key questions about the distribution of matter in the outer solar system, the processes by which the Sun interacts with the galaxy, and the nature and properties of the nearby galactic medium.

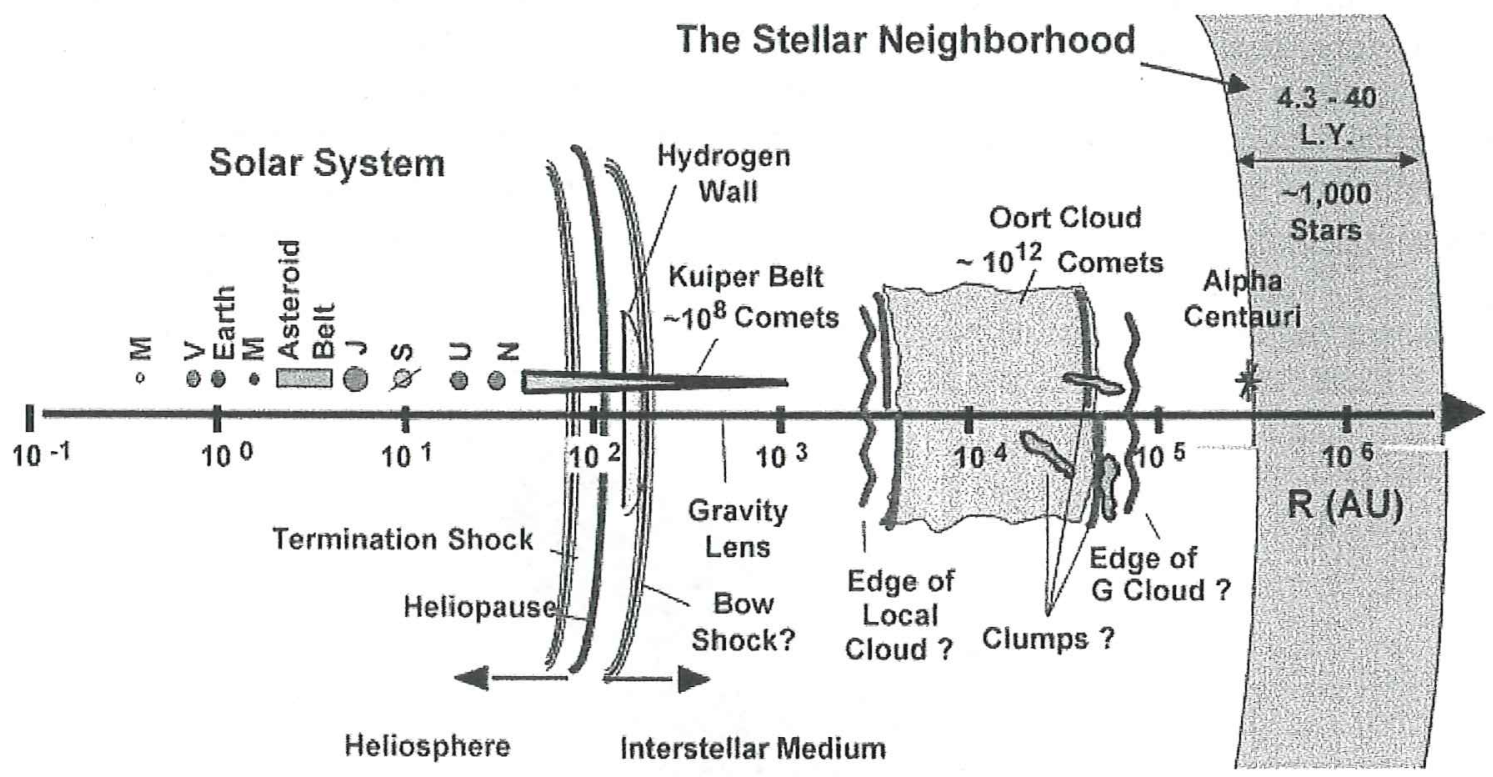

Fig. 2: The local interstellar neighborhood, shown on a logarithmic scale from 0.1 to $10^{6} \mathrm{AU}$.

Figure 2 illustrates the solar system and nearby interstellar medium on a logarithmic scale extending from $<1$ to $10^{6}$ AU. Threaded through the boundaries of the heliosphere is the Kuiper Belt - the source of short period comets. The nearest edge of the low-density interstellar cloud that presently surrounds our solar system is thought to be several thousand AU away. The Oort cloud is a spherical shell of comets extending from $<10,000$ to $\sim 100,000$ $\mathrm{AU}$ that marks the edge of the Sun's sphere of gravitational influence. The best known member of our nearest star system, Alpha Centauri, lies considerably further away at $\sim 300,000$ AU. Interstellar Probe is to be man's first spacecraft to exit the heliosphere and begin the in-situ exploration of the nearby interstellar medium. 
The principal scientific objectives of the Interstellar Probe mission are to:

- Explore the nature of the interstellar medium and its implications for the origin and evolution of matter in our Galaxy and the Universe;

- Explore the influence of the interstellar medium on the solar system, its dynamics, and its evolution;

- Explore the impact of the solar system on the interstellar medium as an example of the interaction of a stellar system with its environment;

- Explore the outer solar system in search of clues to its origin, and to the nature of other planetary systems.

Examples of the scientific issues that could be addressed are described below, abstracted from earlier discussions in Liewer et al. (2000), and Mewaldt and Liewer (2000).

\subsection{The Nearby Interstellar Medium}

Our Sun is surrounded by a low-density cloud $\left(\sim 0.3 \mathrm{~cm}^{-3}\right)$, often referred to as the local interstellar cloud (LIC). Direct observations of our LIC, shown schematically in Figure 3, will provide a unique opportunity to derive the physical properties of a sample of interstellar material, free from uncertainties that result from the exclusion of plasma, small dust particles and low-energy cosmic rays from the heliosphere, and free from uncertainties that plague the interpretation of astronomical data. Measurements will be made of the elemental and isotopic composition of the ionized and neutral components of the interstellar gas and of low-energy particle components, and of the composition and size distribution of interstellar dust (see Figure 4). These measurements will provide a benchmark for comparison with solar system abundances (representing the presolar nebula) and with abundances from more distant galactic regions, thereby providing constraints on galactic chemical evolution theories.

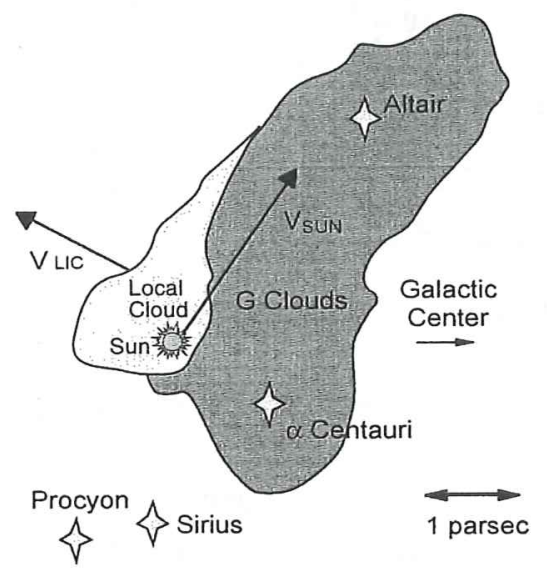

Fig. 3: Map of our local galactic neighborhood showing the Sun located near the edge of our local cloud. Alpha Centauri is 4.3 light-years away in the neighboring G-cloud complex. Outside of these clouds the density may be $<10^{-3}$ atoms $/ \mathrm{cm}^{3}$. (Illustration by P. Frisch)

The properties of the magnetic field in the ISM and in the region beyond the termination shock are essentially unknown. Interstellar Probe will make the first in situ measurements of interstellar magnetic fields and of the density, temperature, and ionization-state of the interstellar gas. It will also of measure the spectra and composition of cosmic ray nuclei and 
electrons, beyond the influence of the heliosphere, and investigate processes that include acceleration by supernova shock waves, interstellar radio and x-ray emission, recent nucleosynthesis, and the heating and dynamics of the ISM.
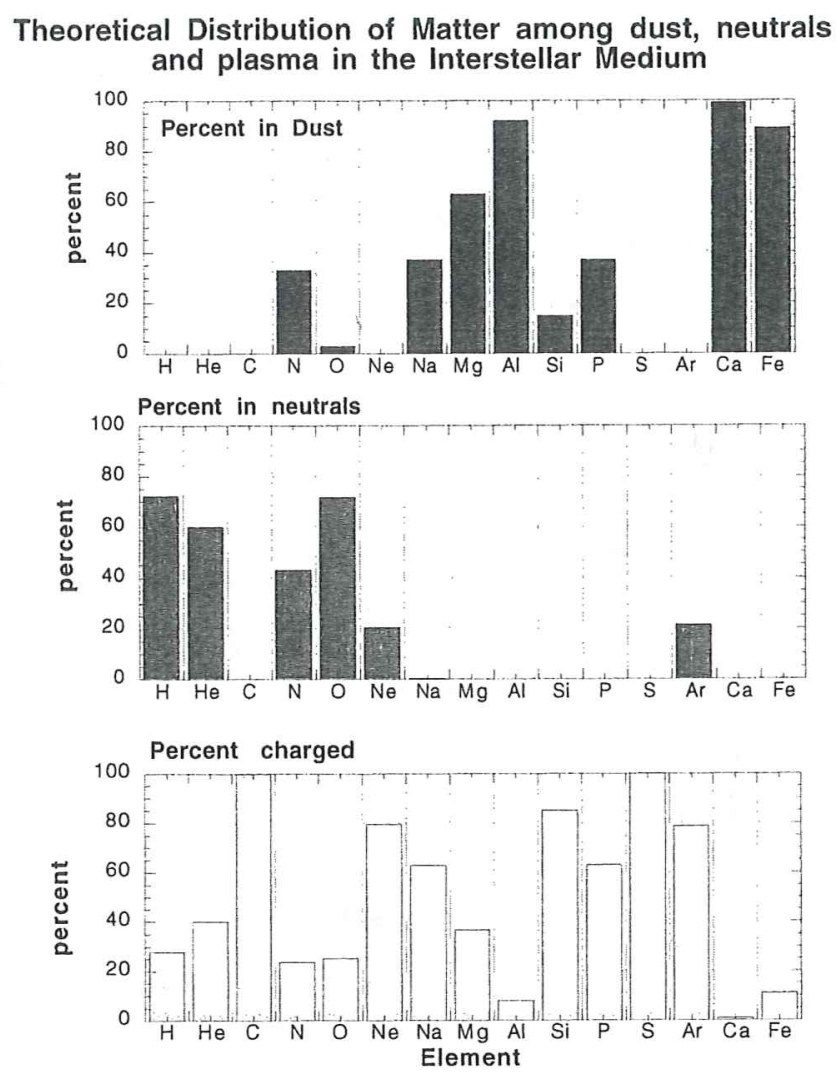

Figure 4: Theoretical model of the distribution of the elements dust, neutral, and ionized states in the local interstellar cloud (data from P. Frisch, personal communication, 1999).

The cosmic infrared radiation background (CIRB) is the integrated light from all stars and galaxies that cannot be resolved into individual objects. Its spectrum provides information on the era when the first stars formed and element nucleosynthesis began. The Cosmic Background Explorer (COBE) established limits on the energy released by stars since the beginning of time by measuring the CIRB at wavelengths $>140$ microns (Hauser et al. 1998), but shorter wavelengths could not observed because of the very bright foreground emission from zodiacal light. The zodiacal dust decreases with heliocentric radius and beyond $10 \mathrm{AU}$ Interstellar Probe may be able to detect the CIRB at wavelengths $<140$ microns.

\subsection{The Interaction between the Interstellar Medium and the Solar Wind}

As the solar wind streams outward through the solar system, it interacts with the ISM to create the heliosphere (see Figure 1). The size of the heliosphere is determined by a balance between solar-wind ram pressure and interstellar pressure. Voyager 1, now just beyond 80 AU, will soon reach the first boundary in this complex structure, the solar-wind termination shock. Beyond this lies the heliopause - the boundary between solar wind and interstellar plasma. The Voyagers have detected radio emissions apparently caused by interplanetary shocks hitting denser interstellar plasma just beyond the heliopause (Gurnett et al. 1993; 
McNutt et al. 1995). Voyager 1 will provide a first direct test of our current understanding of heliospheric structure, although some Voyager instruments were designed for other purposes than to explore the boundaries of the heliosphere and ISM. Interstellar Probe's enhanced capabilities and lifetime will greatly extend Voyager's exploratory studies.

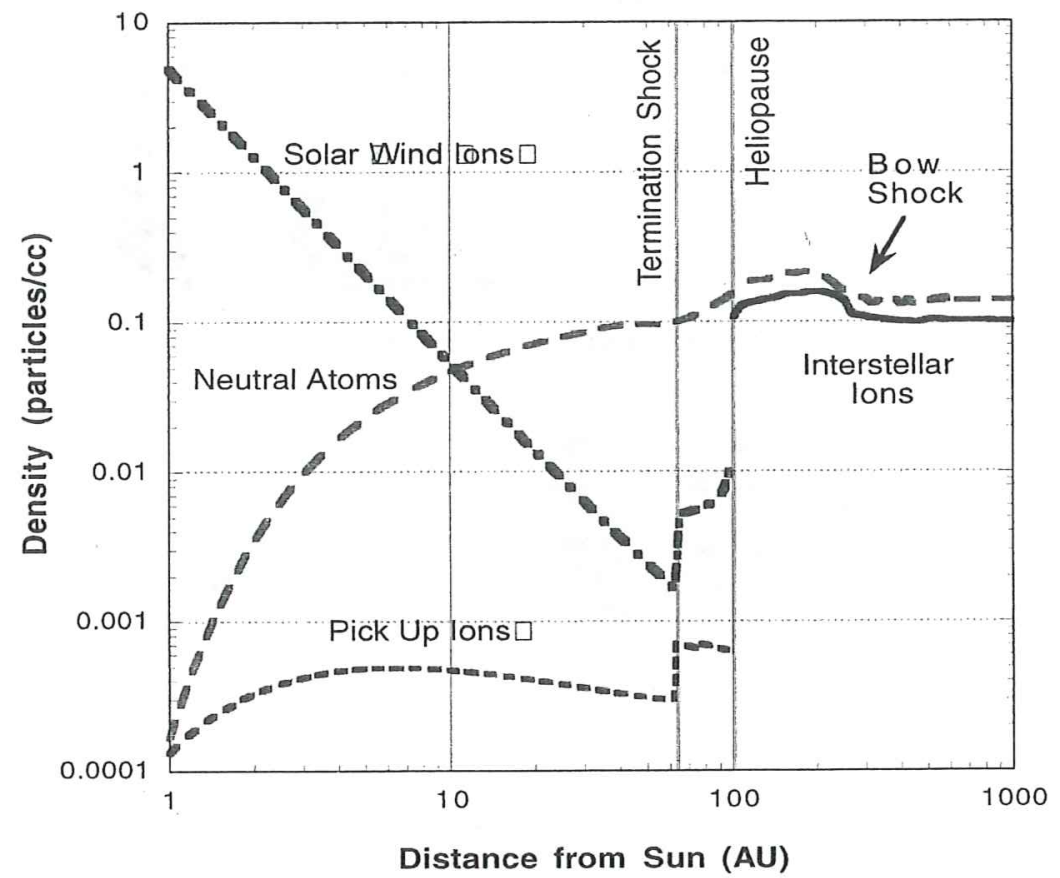

Figure 5: Plasma and neutral-particle densities computed by a hydrodynamic model are plotted as a function of distance from the Sun (courtesy of G. Zank).

The termination shock is a powerful accelerator with particle energies reaching as high as 1 $\mathrm{GeV}$. In situ studies of shock structure, plasma heating, and acceleration processes at the termination shock will serve as a model for other astrophysical shocks. Beyond the termination shock, in the heliosheath, the solar wind flow is turned to match that of the diverted interstellar plasma (see Figure 1). There may be a bow shock created in the interstellar medium ahead of the nose of the heliosphere, depending on the unknown interstellar magnetic field strength. Energetic ions created by charge exchange in the heliosheath can be used to image the 3-D structure of the heliosphere. Charge-exchange collisions cause a pile-up of neutral hydrogen at the heliosphere nose, referred to as the "hydrogen wall" (see, e.g., Zank 1999). Interstellar Probe will pass through these boundary regions and make in situ measurements to answer questions regarding the size, structure and dynamics of the heliosphere and processes occurring at the boundaries. Our heliosphere will serve as an example of how a star interacts with its environment.

\subsection{The Outer Solar System}

Some 4.5 billion years ago our solar system condensed out of the ISM from a protoplanetary disk nebula. Interstellar Probe can address key questions having to do with the radial extent of the primordial solar nebula (see Figure 6) by measuring the radial variation of the number of small bodies in the Kuiper Belt, or, less directly, by measuring the distribution of dust grains derived from Kuiper Belt objects. Interstellar Probe will provide in situ and remote sensing surveys of both interplanetary and interstellar dust in the heliosphere and the 
VLISM, determining mass and orbital distributions as well as composition. Understanding the Kuiper Belt will aid the interpretation of planet-forming disks in other stellar systems.

It may also be possible to investigate the nature and evolution of organic material in the outer solar system and LISM. There is organic material in our solar system (in asteroids, comets, meteorites and dust) and in the ISM. Amino acids have been found in meteorites, but it is not known if they exist in the ISM. Organic materials from both small bodies and the ISM are known to reach Earth and may have played a role in the emergence of life on our planet (see, e.g., Pendelton and Tielens 1997). The development of instrumentation to make such measurements is one of the challenges for the ISP payload.

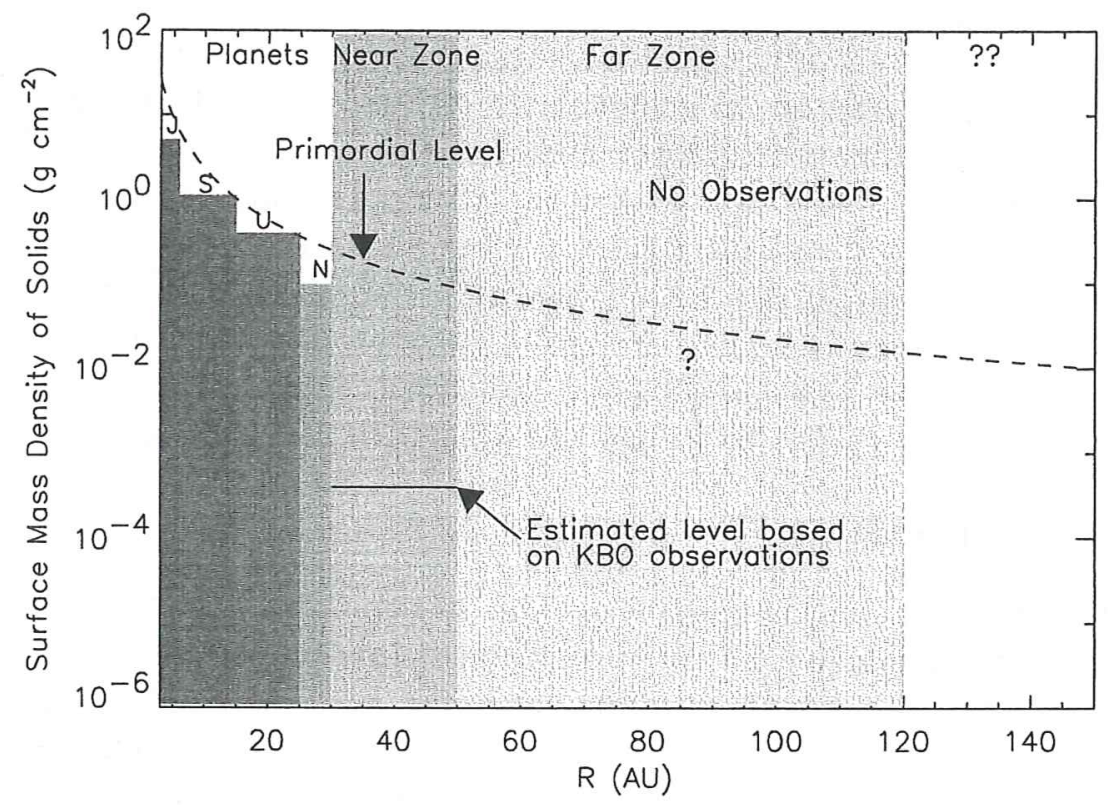

Figure 6: The mass density of the solar system as a function of distance from the Sun. The decrease in density from $\sim 30$ to $50 \mathrm{AU}$ may be due to the scattering of material by the giant planets. Beyond 50 AU there are no observations and the density may increase.

\section{MISSION REQUIREMENTS AND CONCEPT}

The mission requirements for Interstellar Probe are driven by the need to accomplish the scientific goals on a reasonable schedule within the resources likely to be available for such a mission. The following requirements were defined by the ISPSTDT after considerable discussion. To meet the scientific goals it is necessary to cross the heliopause and make a significant penetration into the ISM. The ISPSTDT decided that the probe should be designed to reach a minimum distance of $200 \mathrm{AU}$ within 15 years, with a goal of continuing to $\sim 400$ AU. This requires a spacecraft velocity of $\sim 15$ AU/year or more $(\sim 70 \mathrm{~km} / \mathrm{sec})$, several times that of the Voyagers (Voyager-1 $3.6 \mathrm{AU} / \mathrm{yr}$; Voyager-2 3.3 AU/yr). The spacecraft should spin to allow in situ sampling of particle, plasma, and magnetic field distributions and to allow the remote-sensing instruments to scan the sky. The trajectory should aim reasonably close to the nose of the heliosphere to provide the shortest route to the ISM and to benefit studies of in-flowing interstellar plasma and neutral particles. A total of $25 \mathrm{~kg}$ and $20 \mathrm{~W}$ are allocated for the scientific payload. Science and engineering data are to be collected continuously at a bit rate that averages $30 \mathrm{bps}$ out to at least $200 \mathrm{AU}$. 
The mission concept developed by the ISPSTDT and the Mission Design Team at JPL meets these requirements. Reaching $200 \mathrm{AU}$ within 15 years requires advanced propulsion of the options considered, only solar sails and nuclear-electric propulsion (NEP) were judged capable of meeting this requirement within a reasonable development time. Solar-sail propulsion was selected because: a) it is more compatible with the requirement to measure particles and fields continuously in the outer heliosphere (the sail can be jettisoned within a few AU of the Sun, but an NEP system (and its associated backgrounds) must continue for many years to achieve the required $\Delta \mathrm{V}$ ); b) the size and cost of sail systems were judged to be more consistent with guidelines for the SEC Road Map; and c) solar sail propulsion is also of interest for other missions (e.g., Mulligan et al. 2000).

The spacecraft designed by JPL's Mission Design Team has a mass (excluding sail) of $\sim 150 \mathrm{~kg}$ including the instruments. To achieve a $15 \mathrm{AU} /$ year exit velocity, a sail with $1 \mathrm{~g} / \mathrm{m}^{2}$ areal density (sail plus support structure) and a radius of $\sim 200 \mathrm{~m}$ is needed. The total accelerated mass (spacecraft plus sail system) is $246 \mathrm{~kg}$. The spacecraft initially goes in to $0.25 \mathrm{AU}$ to obtain increased radiation pressure before heading towards the nose of the heliosphere. The sail is jettisoned at $\sim 5$ AU when further acceleration from radiation pressure becomes negligible, thereby avoiding potential interference with the instruments. For additional discussion of the mission concept, trajectory, solar-sail propulsion system and spacecraft, see Liewer et al. (2000, 2001) and Mewaldt and Liewer (2000).

\section{SCIENTIIFIC PAYLOAD}

To achieve the broad scientific objectives of this mission, the strawman scientific payload (Table 1) includes an advanced set of miniaturized, low-power instruments specifically designed to make comprehensive measurements in the outer heliosphere and nearby ISM, with capabilities generally far superior to the Voyager instruments. Table 1 summarizes the strawman payload selected by the ISPSTDT. The mass and power estimates for these instruments assume substantial development in the coming years to achieve greater integration of electronics, to reduce the size of power supplies, and to optimize sensor design and packaging. We summarize briefly some of the key capabilities of this payload, including instances where new technology can be a benefit.

TABLE 1. Strawman Instrument Payload

\begin{tabular}{|ll|}
\hline Instrument & Additional Candidates \\
Magnetometer & Kuiper Belt Imager \\
Plasma and Radio Waves & New Concept Molecular \\
& $\begin{array}{l}\text { Analyzer } \\
\text { Suprathermal Ion Charge- } \\
\text { Star Wind/Interstellar Plasma/Electrons }\end{array}$ \\
& Cosmic Ray Antiprotons \\
Pickup and Interstellar Ion Composition & \\
Interstellar Neutral Atoms & \\
Suprathermal Ions/Electrons & Resource Requirements \\
Cosmic Ray H, He, Electrons, Positrons & - Mass: $25 \mathrm{~kg}$ \\
Anomalous \& Galactic Cosmic Ray & Bit Rate: 25 bps \\
Composition & - Power 20 W \\
Dust Composition & \\
Infrared Instrument & \\
Energetic Neutral Atom (ENA) Imaging & \\
UV Photometer & \\
\hline
\end{tabular}




\subsection{In situ Measurements}

Interstellar Probe will encounter a variety of plasma, energetic particle and magnetic field environments, many for the first time in nature, and a comprehensive package of in situ instruments with adequate collecting power, species resolution, and dynamic range is therefore needed. All particles and fields investigations will benefit from a spinning spacecraft. Approximate alignment of the spin axis with the ram direction is the optimum configuration. To scan interstellar distributions using the spacecraft spin, a moderate offset of the ram direction from the ISM inflow direction (by $15^{\circ}$ to $20^{\circ}$ ) may be beneficial.

Vector Magnetometer: The purpose of the magnetometer is to map the magnetic fields in the outer heliosphere and sample for the first time interstellar fields that cannot be detected from Earth. The lowest field strengths are expected to be $\sim 0.01 \mathrm{nT}$, just inside the termination shock. Although the required sensitivity can be achieved with current magnetometers, such weak fields will place challenging requirements on spacecraft electromagnetic cleanliness, and $\mathrm{a} \sim 30 \mathrm{~m}$ boom may be required to negate spacecraft-generated fields. Two magnetometers are envisioned, one midway along the boom and the other at the end. The spacecraft spin rate of $\sim 6 \mathrm{rpm}$ will aid in correcting for spacecraft generated fields.

Electric Fields and Radio: There are three high priority objectives for the plasma wave investigation: (1) Monitor low-frequency heliospheric radio emissions apparently caused by large interplanetary shocks (see Gurnett et al. 1993). This requires a frequency range up to $\sim 5$ $\mathrm{kHz}$ and $\sim 10$ times the sensitivity of Voyager. (2) Survey plasma waves in the outer heliosphere and interstellar medium. (3) Provide an accurate, independent determination of the plasma density in low-density regions such as the outer heliosphere, where such measurements are difficult with a plasma instrument. An instrument which meets the above objectives could be composed of a single dipole antenna of $>100 \mathrm{~m}$ tip-to-tip, a preamplifier, and a single $5-\mathrm{kHz}$ wideband receiver. The payload processor would perform Fourier transforms to provide spectral information and average spectra, find peaks (as a means of identifying bursty plasma waves) and perform data compression.

Solar Wind, Pickup Ion and Interstellar Plasma: The plasma instrumentation on the Interstellar Probe will map the solar wind up to the termination shock, follow its thermalization in the heliosheath, and detect the transition from solar to interstellar plasma at the heliopause. It should also determine the composition and velocity distribution of interstellar pickup ions and the interstellar plasma itself.

Electron and Ion Sensors: The plasma instrument should include an ion sensor to measure beam-like plasmas in the sunward and anti-sunward directions and an electron sensor to measure core and halo electrons over $\sim 4 \pi$. These sensors need to measure plasma temperature, density, speed, and pressure at intensities considerably lower than typical solar wind conditions. The energy range should exceed $10 \mathrm{keV} / \mathrm{Q}$ and the geometric factor should be $\geq 10^{-2} \mathrm{~cm}^{2}$ to allow good sensitivity for heavy ions. Because interstellar plasma is relatively cold $\left(\sim 10^{4} \mathrm{~K}\right)$, an energy per charge (E/Q) measurement will result in good mass per charge $(\mathrm{M} / \mathrm{Q})$ resolution. Suprathermal electron measurements (and the implied magnetic topology) will provide information on the nature of the termination shock and heliosheath. Due to telemetry limitations, pitch-angle distributions may be computed on board.

Pickup-Ion and Interstellar-Plasma Distribution and Composition Sensor: This sensor will determine the elemental and isotopic composition of the LISM by observing interstellar pickup ions inside the heliosphere and interstellar plasma beyond the heliopause, including the isotopes of key refractory elements such as $\mathrm{C}, \mathrm{Mg}, \mathrm{Si}, \mathrm{Ca}$, and Fe. Science objectives include the ionization-state of the LISM and the nuclear and chemical history of interstellar 
material. In addition, this instrument will provide the source composition of ions accelerated at the termination shock. While only moderate mass resolution is needed for elemental composition and ionization-state studies, a mass resolution of $M / \Delta M \geq 40$ is required for isotope measurements. The opposing flow directions of pickup ions and interstellar plasma can be covered with separate sensors or with a top-hat design. Conventional time-of-flight sensors are adequate for elemental composition studies. Isotope resolution can be achieved with an isochronous time-of-flight instrument with $360^{\circ}$ field-of-view. To provide these capabilities within the resources indicated in Table 1 will require developments in electronics, high-voltage supplies and sensor materials.

Interstellar Neutral Instrument: The objectives of the interstellar neutral sensor are to provide the density, flow direction, and temperature of interstellar $\mathrm{H}, \mathrm{O}$ and $\mathrm{C}$, and possibly He. In addition, resolution of deuterium would provide the important interstellar $\mathrm{D} / \mathrm{H}$ ratio. These parameters will vary along the flight path from the heliosphere through the heliosheath, heliopause, and hydrogen wall, and on into the undisturbed ISM. While He enters unimpeded, $\mathrm{H}$ and $\mathrm{O}$ are affected by resonant charge exchange in the heliospheric interface region, and a key objective is to determine directly the $\mathrm{H}$ and $\mathrm{O}$ depletion along the trajectory through the hydrogen wall. The ionization-state of the VLISM will be determined by combining neutral and ionized abundances of key species such as $\mathrm{H}, \mathrm{O}, \mathrm{C}$ and He. These capabilities can be achieved by a sensor based on neutral to negative-ion conversion during reflection off a suitable conversion surface, combined with electrostatic deflection and acceleration, and subsequent time-of-flight analysis. Suitable surfaces are under study but require further development.

Suprathermal Ions and Electrons: The suprathermal ion and electron sensors cover the energy range above the plasma regime where particles are accelerated out of the bulk distribution. Important objectives include measuring the injection and acceleration of pickup ions at the termination shock, the suprathermal extension of heated solar-wind and pickup-ion distributions in the heliosheath, and searching for new particle components beyond the heliosphere. The electron sensor will survey electron acceleration in the heliospheric boundary region. To fulfill these objectives requires overlap with the plasma instrument at lower energies and the cosmic ray sensors at higher energies. A $4 \pi$ angular acceptance is important to measure the expected distributions. To differentiate source populations elemental discrimination is necessary (at least $\mathrm{H}, \mathrm{He}, \mathrm{C}, \mathrm{O}, \mathrm{Ne}, \mathrm{Ar}, \mathrm{Fe}$ ). Of these species $\mathrm{H}, \mathrm{He}, \mathrm{O}, \mathrm{Ne}$ and Ar have a high ionization potential, while Fe has a low ionization potential and might originate from other sources.

Cosmic Ray H, He, and Electron Sensor: This instrument will measure the energy spectra of cosmic ray protons, ${ }^{3} \mathrm{He}$, and ${ }^{4} \mathrm{He}$ with energies from $\sim 1$ to $300 \mathrm{MeV} /$ nucleon, where they are excluded from the heliosphere by the solar wind. It should also measure cosmic ray electrons from $\sim 1$ to $100 \mathrm{MeV}$ and identify positrons over a more limited range for comparison with radio and gamma ray measurements of interstellar electrons. Because of dynamic range considerations it is best to measure these light species in a separate instrument from heavier elements (see below). Note that this energy range includes anomalous $\mathrm{H}$ and $\mathrm{He}$ that are accelerated at the termination shock, as well as cosmic ray ${ }^{1} \mathrm{H},{ }^{3} \mathrm{He}$, and ${ }^{4} \mathrm{He}$ in an energy range that is not accessible at $1 \mathrm{AU}$. One concept is described in Dröge et al (2001).

Anomalous and Galactic Cosmic Ray Composition: Measurements of the composition of anomalous and galactic cosmic rays from $\sim 1$ to $\sim 300 \mathrm{MeV} /$ nucleon can address key questions about the acceleration of particles at the termination shock and at supernova shock waves, the transport of particles in the ISM, and the origin of the accelerated material. To discriminate 
between suggested sources of ACR ions and acceleration models requires measurements of abundant $\mathrm{ACR}$ species ( $\mathrm{He}, \mathrm{C}, \mathrm{N}, \mathrm{O}, \mathrm{Ne}$, and $\mathrm{Ar}$ ) as well as rare species $(\mathrm{C}, \mathrm{Mg}, \mathrm{Si}, \mathrm{S}$, and possibly $\mathrm{Fe}$ ) whose origin is controversial. Isotopic studies of $\mathrm{C}, \mathrm{O}, \mathrm{Ne}$, and $\mathrm{Ar}$ will provide key information for understanding the origin and evolution of neutral interstellar material, complementing studies at plasma energies. Other objectives are to measure the composition and energy spectra of interstellar cosmic rays with energies that are excluded from the heliosphere by the solar wind ( $<300 \mathrm{MeV} /$ nucleon), and searching for evidence of nearby galactic sources. Measurements of radioactive clocks will distinguish cosmic ray acceleration and transport models. To minimize resource requirements, a double-ended telescope could measure both anomalous and galactic cosmic ray elemental and isotopic composition from $\mathrm{Li}$ to $\mathrm{Ni}(3 \leq \mathrm{Z} \leq 28)$ with energies from $\sim 2$ to $\sim 300 \mathrm{MeV} /$ nucleon. Although such instrumentation can be built with current technology, there is a need for the development of low-power electronics for pulse-height-analysis and thin, large-area solid-state detectors.

Dust Distribution and Composition Instrument: The objectives of this instrument are to determine the flux, mass distribution, and elemental composition of dust particles in the interstellar upwind direction with varying distance from the Sun. The expected rate of large interstellar grains is $\sim 150$ particles per year (for $100 \mathrm{~cm}^{2}$ detection area and $>0.4$ micron particles). The flux of small interstellar grains will exceed this and increase with distance from the Sun. An additional component, with a different impact direction, is expected to produce 500 to 50,000 events/year in the Kuiper Belt. Detectors to measure the flux and mass distribution of dust particles are available; elemental composition detectors exist as prototype versions. Test facilities covering the range of expected impact velocities ( 75 to $100 \mathrm{~km} / \mathrm{sec}$ ) are needed.

\subsection{Imaging Instruments}

Energetic Neutral Atom Imager: This instrument will measure angular and energy distributions of energetic neutral atoms (ENAs) born in the heliosheath by charge exchange between hot post-shock solar-wind protons (including pickup ions) and interstellar neutral gas. Measurements from separated points will allow reconstruction of the 3-D structure of the ENA source region. The nature of the termination shock can also be investigated: a stronger shock will result in hotter post-shock solar-wind plasma and higher ENA energies. These measurements can also probe the fate of pickup protons at and beyond the termination shock and reveal details of the acceleration of anomalous particles. Simulations show that an energy range of $\sim 0.3-7 \mathrm{keV}$, a resolution of $\Delta \mathrm{E} / \mathrm{E} \leq 1$, and an angular resolution of $\sim 70 \times 70$ is adequate for these questions. ENAs can be converted in an ultra-thin foil, analyzed with an electrostatic analyzer, and then detected using a triple coincidence scheme. To generate a 3-D image of the termination shock, at least two view angles are required. Slices of ENA emission will be recorded as the spacecraft moves from the inner heliosphere to beyond the termination shock.

$U V$ Imager: The ultraviolet imager will measure the glow of interstellar atomic hydrogen under solar illumination in the Ly $\alpha 121.6-\mathrm{nm}$ resonance line. The main goal is to establish an accurate determination of the absolute number density of atomic hydrogen in the LISM. The mean free path of Ly $\alpha$ photons is $10-20 \mathrm{AU}$ in $\mathrm{H}$ with a density of $\sim 0.1 \mathrm{~cm}^{-3}$. While exiting the heliosphere the density profile and thickness of the "hydrogen wall" can be mapped. After leaving the hydrogen wall the galactic Ly $\alpha$ background can be measured for the first time. The instrument can be simple and based on proven technology. A broadband (10 nm) 
photometer centered on $121.6 \mathrm{~nm}$ with a $\sim 4^{\circ}$ field of view would measure the glow over a swath of the sky as the spacecraft spins. The required sensitivity is $\sim 1$ count/Rayleigh.

IR Imager: The primary scientific objectives of the infrared imager are to measure the radial distribution of dust in the outer solar system and to measure the cosmic infrared radiation background (CIRB). Such measurements are impossible in the inner solar system due to the overwhelming emission of zodiacal dust near the Earth. Both issues can be addressed with unprecedented precision by flying a small telescope to $200 \mathrm{AU}$ with detectors covering a wavelength range from 5 to 150 microns. The strawman design is a $10-\mathrm{cm}$ telescope that feeds a series of detectors covering wavelength bands (ideally) at 5, 12, 25, 60, 100 , and 150 microns. The detectors would scan a roughly stable circle in the sky; zodiacal emission would be separated from the CIRB by watching the brightness at each spot along this circle diminish and finally plateau as the spacecraft leaves the Solar System. Other goals include searching for dust structures associated with planets, asteroids and comets. This instrument concept can benefit from development in several areas, including detector technology and cooling.

\subsection{Potential Payload Enhancements}

Kuiper Belt Imager: This instrument would use a small CCD camera to survey the number of Kuiper Belt objects (KBOs) from 30 to 200 AU. The estimated mean separation of 1-km $\mathrm{KBO}$ s between 30 and $50 \mathrm{AU}$ is $\sim 0.02 \mathrm{AU}$, while for $10-\mathrm{km}$ KBOs the mean separation is $\sim 0.1$ AU (R. Malhotra, personal communication). At larger distances no reliable estimates are available, but theoretical speculations include the possibility that the density decreases, remains constant, or increases by 1 to 2 orders of magnitude. With the CCD camera scanning the sky at $90^{\circ}$ to the spin axis, KBOs can be identified by comparing images of the same field taken on three successive rotations. The expected number of detected objects ranges from $\sim 10^{4}$ if the number density remains constant, to $\sim 30$ times as many if the number density increases by a factor of 50 beyond $70 \mathrm{AU}$. This experiment can easily distinguish the various theoretical possibilities.

In situ sampling of organic material: During the Comet Halley encounters it was demonstrated that elemental abundances of dust grains can be determined by impacting a target (e.g. silver) followed by subsequent time-of-flight spectroscopy triggered by the impact flash. This led to the discovery of "CHONs" - organic dust particles and coatings of mineral grains (Formenkova et al. 1994). Further investigations have shown that several molecular structures can be inferred from the measured abundance ratios of $\mathrm{C}, \mathrm{H}, \mathrm{O}$ and $\mathrm{N}$. The goal of elucidating molecular structure and identifying molecular families of complex organic species in the outer solar system and ISM is of intense interest, but indeed formidable. Several groups are pursuing experimental techniques that might make this possible.

Ionic Charge-State Analyzer: It would be very desirable to include an instrument to measure the mean charge-state of suprathermal nuclei. This would distinguish unambiguously between possible source populations accelerated at the termination shock (and elsewhere), including interstellar pickup ions (singly-charged species with high first ionization potential), "inner source" pickup ions (mostly singly-charged refractory ions), re-accelerated solar wind ( $\sim 1$ MK charge states), and galactic cosmic rays (fully stripped). However, charge-sensitive instruments typically require significant resources to achieve an adequate geometry factor. As a result, higher priority was given to a sensor with greater collecting power, angular acceptance, and only elemental composition capability. 
Low-Energy Cosmic-Ray Antiproton Measurements: The detection of exotic sources of low-energy $(<300 \mathrm{MeV})$ antiprotons in interstellar space could have significant astrophysical and cosmological implications. Proposed sources include the annihilation and decay of dark matter in the galactic halo (Bergström et al. 1999) and evaporation of primordial black holes (Hawking radiation; see, e.g., Maki, Mitsui, and Orito 1996). It is unlikely that these lowenergy antiprotons will be observable inside the heliosphere because they are excluded by the solar wind. A small instrument to identify antiprotons could be based on measuring the kinetic energy $(\sim 2 \mathrm{GeV})$ released in the form of pions during antiproton-proton annihilation. One concept (A. Mosiev et al., personal communication) would use a time-of-flight system to measure the velocity of incident particles and a calorimeter to measure the annihilation energy. Exotic sources of antiprotons would be identified by their unique energy spectra.

\section{SUMMARY}

Although most of the instruments required for this mission have considerable flight heritage and could be built today, all would benefit from new technology in order to optimize the scientific return within the very restrictive weight and power resources. In addition, exciting instrument concepts such as the molecular analyzer will require considerable development. The most critical technology needed to carry out the mission described here is solar sail propulsion. Although solar sails have been studied extensively, (e.g., Wright 1992), they have never flown in space. Indeed, to achieve the spacecraft velocities envisioned here will require rather advanced sails that will have to be tested in one or more flight demonstrations (e.g., Wallace 1999). Fortunately, several other missions can also benefit from solar-sail propulsion (see, e.g., Mulligan et al. 2000). If this program is successful, launch could be as early as 2010, and Interstellar Probe can serve as the first step in a more ambitious program to explore the outer solar system and nearby galactic neighborhood.

\section{ACKNOWLEDGEMENTS}

A portion of this work was conducted at Caltech's Jet Propulsion Laboratory under contract with NASA. We gratefully acknowledge the help of E. Danielson, JPL; R. Forward, Forward Unlimited; T. Linde, U. of Chicago; J. Ormes, GSFC; M. Ressler, JPL; T. Wdowiak, U. of Alabama at Birmingham; M. Wiedenbeck, JPL; the JPL Advanced Project Design Team (Team X), led by R. Oberto; and additional members of the JPL Interstellar Program: S. Dagostino, K. Evans, W. Fang, R. Frisbee, C. Gardner, H. Garrett, S. Leifer, R. Miyake, N. Murphy, B. Nesmith, F. Pinto, G. Sprague, P. Willis, and K. Wilson.

\section{REFERENCES}

Bergstrom, L., Edsjö, J., and Ulliö, P., Astrophys. J., 526, 215, 1999.

Burch, J. L., et al., Sun-Earth Connection Roadmap - Strategic Planning for the Years 2000$2020,1996$.

Burke, B., et al., Report of the Astronomy and Astrophysics Task Group (B. Burke, chair), in

Space Science in the 21st Century - Imperatives for the Decade 1995-2015, National Academy of Sciences, 1988.

Dröge, W., Heber, B., Potgieter, M. S., Zank, G. P., and Mewaldt, R. A., "A Cosmic Ray Detector for an Interstellar Probe" these proceedings, 2001.

Etchegaray, M. I., Preliminary Scientific Rationale for a Voyage to a Thousand Astronomical Units, JPL Publication 87-17, 1987. 
Formenkova, M. N., Chang, S., and Mukhin, L. M., Geochim Cosmochimica Acta, 58, No. 20, 4503-4512, 1994.

Gurnett, D. A., Kurth, W. S, Allendorf, S. C., and Poynter, R. L., Science, 262, 199, 1993.

Hauser, M. G., et al., Astrophys. J. 508, 25, 1998.

Holzer, T. E., Mewaldt, R. A., and Neugebauer, M., The Interstellar Probe: Scientific Objectives and Requirements for a Frontier Mission to the Heliospheric Boundary and Interstellar Space, Report of the Interstellar Probe Workshop, Ballston, VA, 1990.

Jaffe, L. D., et al., An Interstellar Precursor Mission, JPL Publication 77-70, 1977

Jaffe, L. D., and Ivie, C. D., Icarus, 39, 486, 1979.

Liewer, P. C., Mewaldt, R. A., Ayon, J. A., and Wallace, R. A., "NASA's Interstellar Probe Mission", in Space Technology and Application International Forum-2000, edited by M. S. El-Genk, AIP Conference Proceedings CP504, American Institute of Physics, New York, p. 911, 2000.

Liewer, P. C., Mewaldt, R. A., Ayon, J. A., Garner, C., Gavit, S., and Wallace, R., An Interstellar Probe using a Solar Sail: Conceptual Design and Technological Challenges, these proceedings, 2001.

McNutt, R. L. Jr., Lazarus, A. J., Belcher, J. W., Lyon, J., Goodrich, C. C., and Kulkarni, R., Adv. Space Res. 16, 103, 1995.

Mewaldt, R. A., and Liewer, P. C., "An Interstellar Probe Mission to the Boundaries of the Heliosphere and Nearby Interstellar Space", submitted to the AIAA Space 2000 Conference, 2000.

Mewaldt, R. A., Kangas, J., Kerridge, S. J., and Neugebauer, M,. "A Small Interstellar Probe to the Heliospheric Boundary and Interstellar Space", Acta Astronautica, 35 Suppl., 267, 1995.

Maki, K., Mitsui, K., and Orito, S., Phys. Rev. Letters 76, 3474, 1996.

Mulligan et al., "Solar Sail Development and its Implications for Space Weather", submitted to the AIAA Space 2000 Conference.

Neugebauer, M, et al., A Science Strategy for Space Physics, Report of the Committee on Solar and Space Physics and the Committee on Solar Terrestrial Research, M. Neugebauer, Chair, National Academy of Sciences, 1995.

Nock, K. T., "TAU - A Mission to a Thousand Astronomical Units", $19^{\text {th }}$ AIAA/DGLR/JSASS International Electric Propulsion Conf., Colorado Springs, 1987.

Pendelton, Y. J., and Tielens, A. G. G. M., From Stardust to Planetesmals, ASP Conference Series, Volume 122, 1997.

Scarf, F. et al., "The Report of the Solar and Space Physics Task Group" (F. Scarf, chair) in Space Science in the 21st Century - Imperatives for the Decade 1995 - 2015, National Academy of Sciences, 1988.

Stone, E. C., and Cummings, A. C., $26^{\text {th }}$ Internat. Cosmic Ray Conf. (Salt Lake City), 7, 500, 1999.

Strong, K. L. et al., Sun-Earth Connection Roadmap - Strategic Planning for 2000-2025, 1999.

Wallace, R. A., "Precursor Missions to Interstellar Exploration," Proc. IEEE Aerospace Conf., Aspen, CO, Paper 114. 1999.

Wright, J. L., Space Sailing, Gordon and Breach, Amsterdam, 1992.

Zank, G. P., Space Science Reviews, 89(3/4): 413, 1999. 